\title{
Protective Effects of Mizoribine on Cyclosporine A Nephropathy in Rats
}

\author{
SATOSHI HARA, DAISUKE UMINO, TOMONOSUKE SOMEYA, SHUICHIRO FUJINAGA, YOSHIYUKI OHTOMO, \\ HITOHIKO MURAKAMI, AND TOSHIAKI SHIMIZU
}

\begin{abstract}
Department of Pediatrics [S.H., D.U., T.Someya., T.Shimizu], Juntendo University School of Medicine, Tokyo 113-8421, Japan; Divisions of Nephrology [S.F.] and Pathology [H.M.], Saitama Children's Medical Center, Saitama 339-8551, Japan; Department of Pediatrics

[Y.O.], Juntendo University Nerima Hospital, Tokyo 177-0033, Japan
\end{abstract}

\begin{abstract}
The therapeutic benefits of Cyclosporine A (CsA) are often limited by the chronic nephrotoxicity of its long-term use. Chronic nephrotoxicity is manifested by renal function impairment and progressive histopathological kidney lesions characterized by tubular vacuolization, tubular necrosis, interstitial fibrosis, and afferent arteriolopathy. This study tested the hypothesis that the concurrent administration of Mizoribine (MZR) may improve chronic CsA nephrotoxicity. Sprague-Dawley male rats were divided into the following four groups: group 1, control $(n=6)$; group 2, treated with CsA alone $(n=5)$; group 3, treated with CsA and MZR $(n=4)$; and group 4, treated with MZR alone $(n=6)$. The anti-inflammatory and antifibrotic effects of MZR were studied by evaluating the concentrations of the inflammatory mediator, osteopontin, renal function, and histopathology. The interstitial fibrosis was stained blue with Elastica-Massontrichrome and the sections were quantified. The CsA-treated rats showed decreased renal function and increased histologic parameters in comparison with the control rats and also showed significantly increased interstitial fibrosis area and macrophage in comparison with the control rats. The CsA + MZR treatment significantly improved the interstitial fibrosis area and macrophage in comparison with the CsA-treated rats. On the basis of these findings, we suggest MZR effectively attenuates renal macrophage accumulation and the progression of interstitial fibrosis. (Pediatr Res 66: 524-527, 2009)
\end{abstract}

$\mathrm{C}^{\mathrm{x}}$ yclosporine A (CsA), a cyclic peptide extracted from the fungus Tolypocladium inflatum, is an immunosuppressive agent, which was first introduced for the prevention of posttransplant rejection.

Since then, the application of CsA has been extended to the treatment of children with nephrotic syndrome. This has relieved children with steroid dependent and resistant nephrotic syndrome from steroid toxicity (1). However, there is controversy concerning its long-term treatment because of the potential toxicity by chronic CsA nephropathy, which is characterized by tubular atrophy, interstitial fibrosis, and progressive renal impairment (2). Chronic nephrotoxicity is manifested by tubular vacuolization, tubular necrosis, interstitial fibrosis, and afferent arteriolopathy. To date, its early diagnosis is made by

Received February 17, 2009; accepted June 30, 2009.

Correspondence: Satoshi Hara, Ph.D., Department of Pediatrics, Juntendo University School of Medicine, 2-1-1 Hongo, Bunkyo-ku, Tokyo 113-8421, Japan; e-mail: satoshi-h@ y6.dion.ne.jp

DOI: 10.1203/PDR.0b013e3181b9b48a the pathologic findings and no biochemical markers have been established.

On the other hand, Mizoribine (MZR) is a purine nucleotide analog isolated from Eupenicillium brefeldianum (3), which has been used clinically as an immunosuppressant after renal transplantation for lupus nephritis and nephrotic syndrome. Recent studies have demonstrated that MZR suppresses the infiltration of macrophages, which play an important role in the development of interstitial fibrosis and ameliorate the interstitial fibrosis of obstructive nephropathy in rats $(4,5)$. However, its effect has not yet been reported in the CsAtreated rat kidney.

Based on the above findings, MZR may prevent chronic CsA nephropathy. To verify this hypothesis, MZR was administered to CsA-treated rats. This study clearly demonstrated that MZR has synergistic effects on preventing inflammatory process in chronic CsA nephropathy.

\section{MATERIALS AND METHODS}

Animals. Male Sprague-Dawley rats, weighing 200 to 240 g, were housed in cases in a temperature- and light-controlled environment in the Juntendo University Animal Care Facility. Rats received a low-salt diet ( $0.03 \%$ sodium; Test Diet, Richmond, IN). The protocol used in these studies was approved by the Juntendo University Animal Care Committee. ${ }^{1}$

Drugs. CsA (provided by Novartis Pharma, Tokyo, Japan) was diluted in miglyol 812 (Dynamit Nobel AG, Troisdorf, Deutschland) to a final concentration of $30 \mathrm{mg} / \mathrm{mL}$. MZR (Bredinin: Asahi Chemical Industry, Japan) was prepared in physiologic salt solution to a final concentration of $5 \mathrm{mg} / \mathrm{mL}$. The CsA and MZR were given i.p. to the rats before it precipitated. The solutions were freshly prepared and used on the same day.

Experimental groups. The experiment consisted of four groups of 4 to 6 rats: 1) control group: rats received a daily i.p. injection of Migliol 85 (1 $\mathrm{mL} / \mathrm{kg})$ for $8 \mathrm{wk}(n=6)$; 2) CsA group: rats received a daily i.p. injection of CsA (30 mg $/ \mathrm{kg})$ for 8 wk $(n=5) ; 3)$ CsA + MZR group: rats received CsA $(30 \mathrm{mg} / \mathrm{kg})$ and MZR $(5 \mathrm{mg} / \mathrm{kg})$ for $8 \mathrm{wk}(n=4)$; and 4) MZR group: rats received MZR $(5 \mathrm{mg} / \mathrm{kg})$ for $8 \mathrm{wk}(n=6)$.

Basic protocol. Weight-matched rats were randomly assigned to the different treatment groups. Their daily body weight was recorded. At the end of the experiment, blood samples were obtained to evaluate the creatinine, blood urea nitrogen (BUN), and blood CsA trough levels. The rats were killed and their kidneys were removed.

Kidney morphology. Tissue samples were fixed in $10 \%$ (vol/vol) formalin, processed by conventional methods, and embedded in paraffin. Sections were stained with Hematoxylin-Eosin (HE), Periodic Acid Schiff (PAS), Periodic Acid silver-methenamin (PASM), and Elastica-Massontrichrome (E-MT) re-

\footnotetext{
Abbreviations: CsA, Cyclosporine A; E-MT, Elastica-Massontrichrome; HE, Hematoxylin-Eosin; MZR, Mizoribine; OPN, osteopontin; PAS, Periodic Acid Schiff; PASM, Periodic Acid silver-methenamin
} 
agent for the evaluation of interstitial lesions and arteriolopathy. Arteriolopathy of afferent arterioles, especially in the vas afferent was characterized by the expansion of the cell cytoplasm of the terminal arteriolar smooth-muscle cells by eosinophilic, argentaffin material and quantitatively estimated by counting the percentage of arterioles with arteriolopathy per total number of arterioles available for examination with a $200 \times$ objective, with a minimum of 100 arterioles per biopsy assessed. The interstitial fibrosis was stained blue with E-MT, and a color image analyzer quantified the percentage of injured areas (image $\mathbf{J} 1.38$ for Windows by National Institutes of Health).

Immunohistochemistry of ED-1. The detection of infiltrating macrophages relied on immunohistochemistry as follows: the deparaffinized sections from tissues fixed with methanol-Carnoy's solution were stained by the streptavidin-biotin method using an ImmuMark universal immunostaining kit (ICN Immunobiologicals). The following MAb was used as the primary antibody: mouse anti-rat ED-1 (monocyte/macrophage marker, clone ED-1; Serotec). After treatment with $0.3 \%$ hydrogen peroxide to quench endogenous peroxidase and then with endogenous biotin-blocking agent (DAKO) for 20 min each, the sections were incubated with the primary antibody at room temperature for $60 \mathrm{~min}$. The serum of the animals in which the primary antibody had been developed was used as a negative control. Next, biotinylated goat anti-mouse $\mathrm{IgG}$ was used as a secondary antibody for $20 \mathrm{~min}$. The final incubation was carried out for $20 \mathrm{~min}$ with peroxidase-streptavidin conjugate, and positive reactions were visualized by a reaction with 3,3'diaminobenzidine for $5 \mathrm{~min}$. The sections were finally counterstained with $1 \%$ methyl green solution for 20 min. Macrophage infiltration was determined by the number of cells in the cortex that stained positively with the ED-1 antibody. Three randomly chosen nonoverlapping fields $\left(0.29 \mathrm{~mm}^{2}\right.$ each $)$ from the same section of the renal cortex from an individual rat were photographed and the number of cells that were reactive with ED-1 was calculated and averaged (cells $/ 0.29 \mathrm{~mm}^{2}$ ).

Real-time PCR for osteopontin. Real-time quantitative RT-PCR was performed to determine the expression of osteopontin (OPN) in the renal cortex using the Taq Man system according to the manufacturer's protocols (Applied Biosystem). The Taq Man probes were a reporter labeled with fluorescent dye (FAM) on the $5^{\prime}$-end and nonfluorescent glyceraldehydes on the 3 '-end. The expression level of the glyceraldehydes-3-phosphate dehydrogenase (GAPDH) gene was used as an endogeneous control. The amplification and detection of fluorescence was measured using the ABI 7500 system with the following PCR reaction profile: 1 cycle of $50^{\circ} \mathrm{C}$ for $2 \mathrm{~min}$ and $95^{\circ} \mathrm{C}$ for $10 \mathrm{~min}$ and 40 cycles of $95^{\circ} \mathrm{C}$ for $15 \mathrm{~s}$ and $60^{\circ} \mathrm{C}$ for $1 \mathrm{~min}$.

Statistical analysis. The data are reported as the mean \pm SEM (SE). Comparisons between the groups were done by an ANOVA (Tukey's test and Bonferroni/Dunn test). A probability of 0.05 was considered to be statistically significant.

\section{RESULTS}

Changes in body weight and biochemical data. The body weight gain and values for blood creatinine, BUN, and CsA levels are summarized in Table 1. At $8 \mathrm{wk}$, the mean body weight gain of the CsA- and CsA + MZR-treated groups were significantly lower than that of the control $(p<0.01)$. CsA administration significantly increased the serum creatinine and BUN levels $(p<0.01)$. The 8 -wk CsA treatment resulted in an increase in blood CsA concentration to a level of $5400 \pm$ $678 \mathrm{ng} / \mathrm{mL}$, whereas MZR did not influence the level of CsA concentration (CsA + MZR, $6200 \pm 400 \mathrm{ng} / \mathrm{mL} ; p>0.05$ ).

Kidney morphology. The rats treated with CsA had characteristic morphologic findings that were usually evident at 8 wk and were similar to the chronic human CsA renal lesions
(Fig. 1). Focal interstitial fibrosis, tubular atrophy, and mononuclear inflammatory cell infiltration were observed. No tubular vacuolization was found in these specimens in the vas afferent. The histology in the CsA-treated rats showed a typical arteriolopathy. Smooth-muscle cells in the media of the vas afferents were replaced by a PASM positive, a PAS positive, and an orange-G positive material, resulting in a typical circumferential appearance of the lesion; the arteriolopathy was significantly higher in the CsA group than the control group $(48.8 \pm 1.8$ vs $8.3 \pm 1.1 ; p<0.01)$. The addition of MZR $(48.5 \pm 1.7)$ did not decrease the arteriolopathy in comparison with the CsA group ( $p>0.05$; Fig. 2).

There was a significant increase in the percentage of fibrosis areas at the renal cortex in the CsA and CsA + MZR groups in comparison with the control group (CsA, $24.3 \pm 5.4 \%$; $p<$ 0.01 and CsA + MZR, $9.5 \pm 1.1 \% ; p<0.01$, vs control, $1.8 \pm 0.6)$. The concomitant MZR administration with CsA significantly decreased the interstitial damage in comparison with the CsA group ( $p<0.01$; Figs. 3 and 4$)$.

Immunohistochemistry of ED-1. The immunohistochemistry of ED-1 revealed that ED-1-positive cells were minimal in the control group, but their number increased in the CsA group. A quantitative analysis revealed that ED-1-positive cells were significantly increased in the CsA group in comparison with the control group (315.9 \pm 45.4 vs $24.5 \pm 13.4$; $p<0.01)$. The CsA + MZR group $(201.5 \pm 51.3)$ had significantly decreased ED-1-positive cells in comparison with the CsA group ( $p<0.01$; Fig. 5).

Real-time PCR for OPN. RT-PCR analyses were used to assess the expression of OPN mRNA. In the CsA and CsA + MZR groups, there were 6.86-fold and 5.61-fold increases in the OPN mRNA expression, respectively, $(p<0.01$ and $p<$ $0.05)$. In comparison with the control group (2.25-fold), there was no significant difference in the CsA + MZR group in comparison with the CsA group (Fig. 6).

\section{DISCUSSION}

This study demonstrated that MZR treatment significantly improved the histopathological severity of chronic renal damage induced by CsA. These findings suggest that MZR use can provide beneficial effect against chronic CsA nephrotoxicity. In our study, the CsA-treated animals gained less body weight and developed renal insufficiency in comparison with the control animals. This observation is consistent with previous publications under similar experimental conditions $(6,7)$. In fact, there were three deaths (group 2, $n=1$; group 3, $n=2$ ) during the study period. Their data were excluded from the statistical analysis. At the stage of our pilot study, we needed the high dosage and long

Table 1. Body weight gain and biochemical parameter in all the groups

\begin{tabular}{lcccc}
\hline \multicolumn{1}{c}{ Clinical parameter } & Control & CsA & CsA + MZR & MZR \\
\hline BW gain $(\mathrm{g} / 8 \mathrm{wk})$ & $229.1 \pm 46.4$ & $100.8 \pm 13.5^{*}$ & $111.3 \pm 42.5^{*}$ & $178.0 \pm 26.4$ \\
Serum creatinine $(\mathrm{mg} / \mathrm{dL})$ & $0.25 \pm 0.02$ & $0.42 \pm 0.09^{*}$ & $0.36 \pm 0.10$ & $0.24 \pm 0.03$ \\
Serum BUN $(\mathrm{mg} / \mathrm{dL})$ & $14.5 \pm 1.8$ & $41.3 \pm 11.6^{*}$ & $33.1 \pm 8.9^{*}$ & $14.0 \pm 1.9$ \\
Serum CsA $(\mathrm{ng} / \mathrm{mL})$ & & $5400 \pm 678$ & $6200 \pm 400$ & \\
\hline
\end{tabular}

Data are presented as mean value \pm SEM.

$* p<0.01 v s$ control group. 


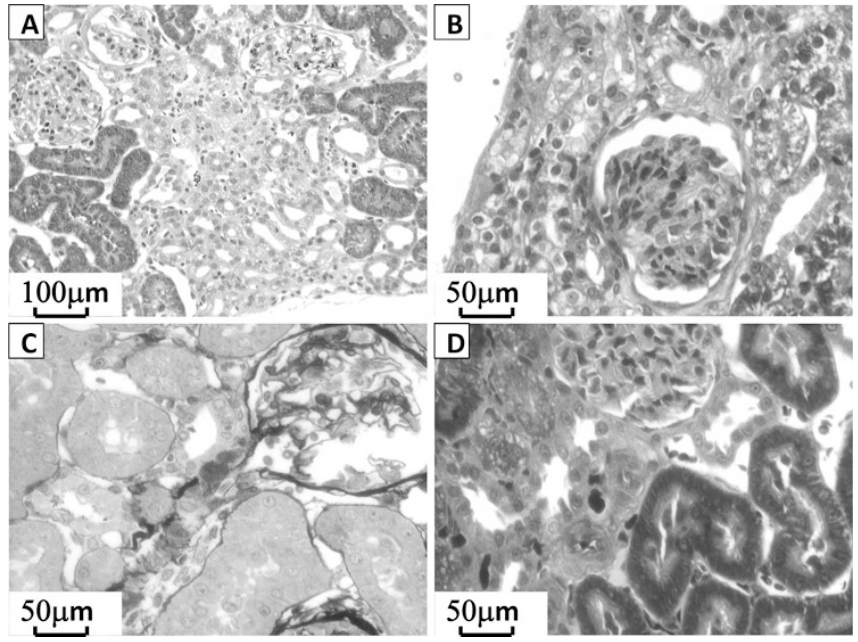

Figure 1. (A) E-MT $(\times 200)$ : CsA treatment for 8 wk induced a typical interstitial fibrosis, inflammatory cell infiltration, and tubular atrophy. $(B)$ EM-T $(\times 400)$ : Arteriole showing hyalinization. $(C)$ PASM $(\times 400)$ : Smooth muscle cells in the media of the vas afferent showing argentaffin. $(D)$ EM-T $(\times 400)$ : Smooth-muscle cells in the afferent glomerular arteriole were replaced by a PAM-positive material, resulting in a typical circumferential appearance of the lesion.

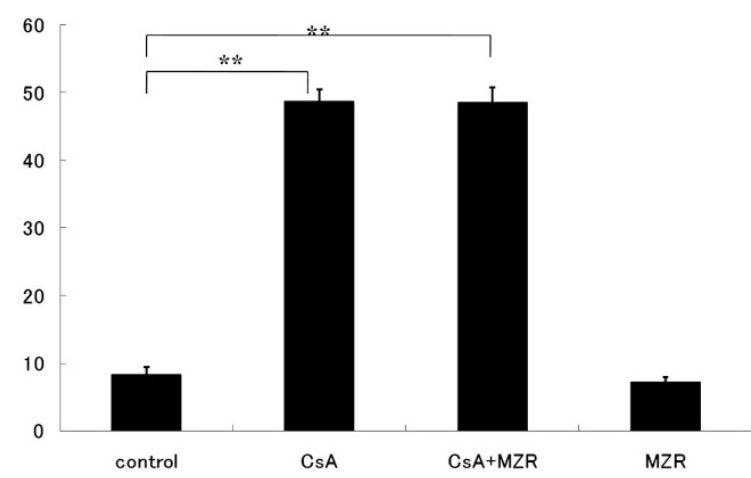

Figure 2. The arteriolopathy was significantly higher in the CsA group than the control group $(p<0.01)$. The addition of MZR did not decrease the arteriolopathy in comparison with the CsA group $(p>0.05)$.
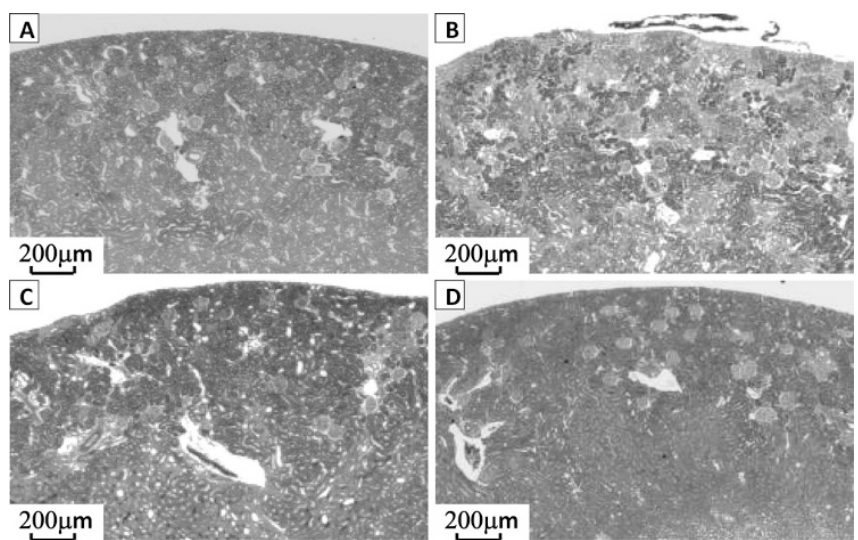

Figure 3. Interstitial fibrosis with E-MT stain. $(A)$ control, $(B)$ CsA-treated, $(C) \mathrm{CsA}+\mathrm{MZR}$ treated, and $(D)$ MZR-treated $(\times 10)$.

period of CsA administration to reproduce CsA nephropathy. High dosage of CsA may affect lethal adverse effect for uremia and over-immunosupression. Renal vasoconstriction, arteriolopathy, and interstitial changes, including inflammation, have all

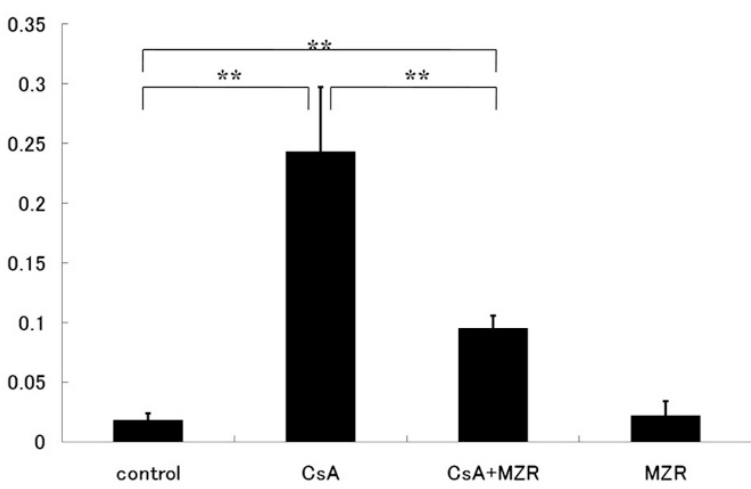

Figure 4. Quantitative analysis of interstitial fibrosis in the experimental groups. Note the significant decrease of interstitial fibrosis with MZR $\left({ }^{*} p<\right.$ $0.01)$.

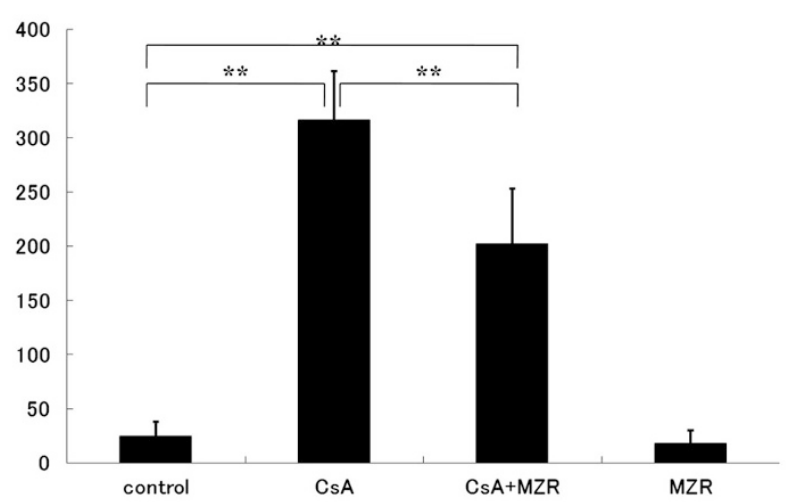

Figure 5. Immunohistochemistry of ED-1. A quantitative analysis revealed that ED-1-positive cells were significantly increased in the CsA group in comparison with the control group (315.9 $\pm 45.4 v s .24 .5 \pm 13.4 ; p<0.01)$. CsA + MZR group $(201.5 \pm 51.3)$ showed a significantly decreased number of ED-1-positive cells in comparison with the CsA group $(p<0.01)$.

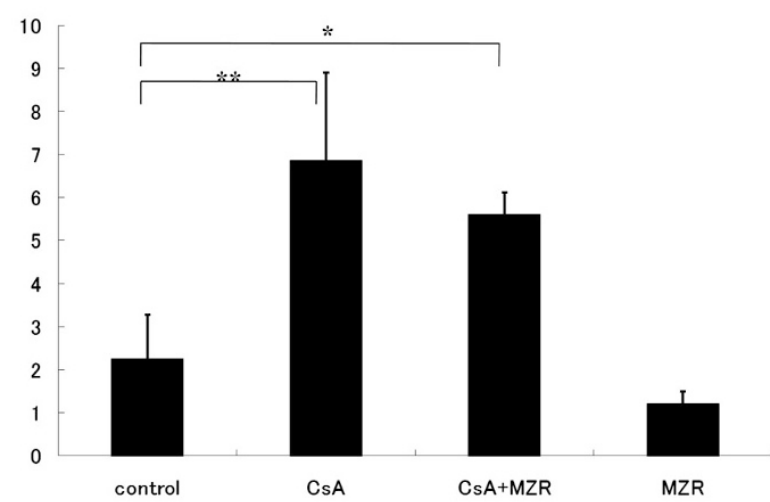

Figure 6. In the CsA and CsA + MZR groups, there were 6.86-fold and 5.61-fold increases, respectively, ( $p<0.01$ and $p<0.05$ ), in the expression of OPN mRNA in comparison with the control group (2.25-fold). No significant difference was seen in the CsA + MZR group in comparison with the CsA group.

been reported to play an important role in CsA renal dysfunction $(8,9)$. The contribution of interstitial damage to CsA nephrotoxicity was further confirmed by the histopathological studies that showed an early loss of tubular segments, increased interstitial matrix deposition, and basement membrane thickening. It has been shown that more than $4 \mathrm{wk}$ CsA administration yields these 
histopathological injuries, whereas CsA administration for shorter periods causes no tubular changes (8). The lesions of chronic CsA nephropathy have been difficult to reproduce in animals. Therefore, the pathogenesis of this type of CsA renal injury has been difficult to study (9). CsA nephrotoxicity has been developed using salt depletion stimulus to accelerate the longterm effects of CsA treatment (10). This study used a model of CsA nephrotoxicity in salt depleted rats. CsA-treated rats showed the characteristic morphologic findings, which were reminiscent of human lesions.

The exact mechanism underlying chronic CsA nephropathy is poorly understood. However, using an animal model, a complex network including the renin-angiotensin system (11), apoptotic cell death (2), cytokines, and chemoattractant molecules (12) has been shown to be involved. Of these, chemoattractants and the resultant inflammatory cell infiltration have been proposed to be important players, because interstitial inflammatory events precede the ongoing fibrosis in chronic CsA nephropathy (13). OPN is a very sensitive indicator of interstitial inflammation, and TGF-beta expression is closely associated with interstitial fibrosis in chronic CsA nephropathy (2).

MZR treatment decreases macrophage infiltration, OPN, and TGF-beta expression and this is associated with improved histopathology, in an animal model of unilateral ureteral obstruction (5). Although, in our study, MZR treatment did not significantly suppress OPN mRNA expression in the rats receiving CsA, a tendency to decrease the OPN level seemed to be accepted. The precise mechanisms by which MZR prevented the progression of CsA nephropathy are not known. However, it could inhibit the up-regulation of chemokines such as OPN, as well as suppress the proliferation of macrophages and lymphocytes.

This finding suggests that MZR is effective in preventing the inflammatory process and interstitial fibrosis in chronic CsA nephropathy.

In summary, this study demonstrated that interstitial fibrosis, resulting from CsA nephropathy, is ameliorated by treatment with the immunosuppressant, MZR. In CsA-treated rats, fibrosis was markedly expanded. The concurrent administration of MZR significantly reduced the expansion of the renal fibrosis. These findings indicate that MZR has beneficial effects on several processes, which contribute to interstitial fibrogenesis, and suggest that MZR therefore has potential for therapeutic use in patients with CsA nephropathy.

\section{REFERENCES}

1. Fujinaga S, Kaneko K, Muto T, Ohtomo Y, Murakami H, Yamashiro Y 2006 Independent risk factors for chronic cyclosporine induced nephropathy in children with nephrotic syndrome. Arch Dis Child 91:666-670

2. Thomas SE, Andoh TF, Pichler RH, Shankland SJ, Couser WG, Bennett WM, Johnson RJ 1998 Accelerated apoptosis characterizes cyclosporine-associated interstitial fibrosis. Kidney Int 53:897-908

3. Mizuno K, Tsujino M, Takada M, Hayashi M, Atsumi K 1974 Studies on bredinin. I. Isolation, characterization and biological properties. J Antibiot (Tokyo) 27:775782

4. Sakai T, Kawamura T, Shirasawa T 1997 Mizoribine improves renal tubulointerstitial fibrosis in unilateral ureteral obstruction (UUO)-treated rat by inhibiting the infiltration of macrophages and the expression of alpha-smooth muscle actin. J Urol 158:2316-2322

5. Sato N, Shiraiwa K, Kai K, Watanabe A, Ogawa S, Kobayashi Y, Yamagishi-Imai H, Utsunomiya Y, Mitarai T 2001 Mizoribine ameliorates the tubulointerstitial fibrosis of obstructive nephropathy. Nephron 89:177-185

6. Yang CW, Ahn HJ, Kim WY, Li C, Jung JY, Yoon SA, Kim YS, Cha JH, Kim J, Bang BK 2003 Synergistic effects of mycophenolate mofetil and losartan in a model of chronic cyclosporine nephropathy. Transplantation 75:309-315

7. Li C, Yang CW, Kim WY, Jung JY, Cha JH, Kim YS, Kim J, Bennett WM, Bang BK 2003 Reversibility of chronic cyclosporine nephropathy in rats after withdrawal of cyclosporine. Am J Physiol Renal Physiol 284:F389-F398

8. Zhong Z, Arteel GE, Connor HD, Yin M, Frankenberg MV, Stachlewitz RF, Raleigh JA, Mason RP, Thurman RG 1998 Cyclosporin A increases hypoxia and free radical production in rat kidneys: prevention by dietary glycine. Am J Physiol 275:F595F604

9. Bennett WM, DeMattos A, Meyer MM, Andoh T, Barry JM 1996 Chronic cyclosporine nephropathy: the Achilles' heel of immunosuppressive therapy. Kidney Int 50:1089-1100

10. Shihab FS, Andoh TF, Tanner AM, Bennett WM 1997 Sodium depletion enhances fibrosis and the expression of TGF-beta1 and matrix proteins in experimental chronic cyclosporine nephropathy. Am J Kidney Dis 30:71-81

11. Yang CW, Ahn HJ, Kim WY, Shin MJ, Kim SK, Park JH, Kim YO, Kim YS, Kim J, Bang BK 2001 Influence of the renin-angiotensin system on epidermal growth factor expression in normal and cyclosporine-treated rat kidney. Kidney Int 60:847857

12. Pichler RH, Franceschini N, Young BA, Hugo C, Andoh TF, Burdmann EA, Shankland SJ, Alpers CE, Bennett WM, Couser WG 1995 Pathogenesis of cyclosporine nephropathy: roles of angiotensin II and osteopontin. J Am Soc Nephrol 6:1186-1196

13. Young BA, Burdmann EA, Johnson RJ, Alpers CE, Giachelli CM, Eng E, Andoh T, Bennett WM, Couser WG 1995 Cellular proliferation and macrophage influx precede interstitial fibrosis in cyclosporine nephrotoxicity. Kidney Int 48:439-448 